\title{
Sites of Synchronous Distant Metastases, Prognosis, and Nomogram for Small Cell Lung Cancer Patients with Bone Metastasis: A Large Cohort Retrospective Study
}

\author{
Zhiyi Fan, ${ }^{1}$ Zhangheng Huang, ${ }^{1}$ Yuexin Tong, ${ }^{1}$ Zhe Zhu, ${ }^{2}$ Xiaohui Huang $\mathbb{D},{ }^{3}$ and He Sun $\mathbb{D}^{1}$ \\ ${ }^{1}$ Department of Spine Surgery, Affiliated Hospital of Chengde Medical University, Chengde, Hebei, China \\ ${ }^{2}$ Department of Hand Surgery, The Second Hospital of Jilin University, Changchun, Jilin, China \\ ${ }^{3}$ Hangzhou Medical College, Hangzhou, Zhejiang, China
}

Correspondence should be addressed to He Sun; sunhejzwk@sina.com

Received 19 March 2021; Accepted 20 July 2021; Published 29 July 2021

Academic Editor: Prasanna Kumar Santhekadur

Copyright (c) 2021 Zhiyi Fan et al. This is an open access article distributed under the Creative Commons Attribution License, which permits unrestricted use, distribution, and reproduction in any medium, provided the original work is properly cited.

Background. Small cell lung cancer (SCLC) is often associated with metastases at the time of diagnosis, and the bone is one of the most common sites. The primary aim of this study was to investigate the site of synchronous distant metastasis to other organs in SCLC patients with bone metastasis (BM) and develop a robust predictive prognostic model. Methods. We retrospectively analyzed the data from patients diagnosed with SCLC with BM in the Surveillance, Epidemiology, and End Results database. Univariate and multivariate Cox analyses were used to identify independent prognostic factors. A prognostic nomogram was constructed and evaluated by calibration curves, receiver operating characteristic (ROC) curves, and decision curve analysis (DCA). Then, according to the sites of metastasis and treatment modality, all patients were stratified into several subgroups. The relationship among sites of metastasis, treatment modality, and overall survival was then analyzed. Results. A total of 6253 patients were included. Independent prognostic factors for SCLC with BM were age, sex, primary site, radiotherapy, chemotherapy, brain metastasis, liver metastasis, and marital status. Calibration, ROC curves, and DCA indicated the excellent performance of the prognostic nomogram. The liver is the most common organ for extraskeletal metastases, followed by the lung. Patients with only BM had the longest mean survival time $(9.30 \pm 0.31$ months). In the subgroup analysis, chemotherapy was an independent prognostic factor for all subgroups. In contrast, radiotherapy showed a positive effect on the prognosis of patients in all subgroups except those with bone and brain metastases and those with bone, lung, and brain metastases. Conclusions. The prognostic nomogram is expected to be an accurate and personalized tool for predicting the prognosis of SCLC patients with BM. Additionally, the determination of the sites of synchronous extraskeletal metastases and the associated prognosis helps in treatment selection.

\section{Background}

Lung cancer is the leading cause of cancer-related death worldwide, and of all the subtypes, small cell lung cancer (SCLC) accounts for approximately $15 \%$ of newly diagnosed lung cancer each year [1,2]. SCLC is the most aggressive type among the lung cancer subtypes and is often accompanied by metastasis at the time of diagnosis $[3,4]$. The incidence of distant metastases at the time of initial diagnosis of SCLC is higher than $60 \%$, and one of the most common sites of metastasis is the bone $[5,6]$. Once bone metastasis (BM) occurs, the risk of skeletal-related diseases increases, leading to a decrease in the patient's quality of life and a poor prognosis [7]. The choice of treatment for BM should be based on the patient's expected survival time [8]. Therefore, predicting the survival time of SCLC patients with BM is of great clinical significance.

Many studies have reported the natural history of patients with BM from nonsmall cell lung cancer [9-12]. However, few reports have been published on the prognostic factors and characteristics of SCLC patients with BM. Gong et al. performed a retrospective analysis of 102 SCLC patients 
with $\mathrm{BM}$ at initial diagnosis and suggested that age, number of BM, and occurrence of distant metastases outside the bone were significant prognostic factors [13]. Notably, the limited sample size and the single-center design are obvious weaknesses of that study. Currently, the TNM staging system is widely used to assess the prognosis of cancer patients [14]. However, in addition to TNM staging, it is well known that clinical characteristics such as sex, age, and treatment modality are important factors that may affect the prognosis of cancer patients $[15,16]$.

To our knowledge, no studies based on large populations to develop a model for predicting the prognosis of SCLC with $\mathrm{BM}$ at initial diagnosis have been performed to date. In addition, the sites of synchronous extraskeletal metastases, such as the lungs, brain, and liver, in SCLC patients with BM at initial diagnosis and the associated prognostic outcomes have not been thoroughly investigated. Therefore, the primary aim of this study was to investigate the sites of synchronous distant metastases and the associated prognosis in SCLC patients with BM at initial diagnosis based on data from the Surveillance, Epidemiology, and End Results (SEER) project and to develop an associated predictive model for prognosis. The second objective was to investigate the survival benefits of the treatment modalities (surgery, radiotherapy, and chemotherapy) by stratifying the patients' metastatic sites and treatment modalities.

\section{Methods}

2.1. Study Population Selection. The workflow of our study is illustrated in Figure 1. This population-based retrospective study used data from the SEER database. The SEER database consists of 18 population-based cancer registries that collect statistical, oncological, diagnostic, and treatment information for approximately $28 \%$ of the population of the United States. This database provides clinical information on cancer patients and greatly facilitates clinical research. Patients diagnosed before 2010 are excluded because the SEER database did not record information on distant metastases (bone, liver, brain, and lung metastases) until 2010. In addition, to ensure adequate follow-up time, patients diagnosed after 2016 are also excluded. Therefore, only SCLC patients diagnosed with BM between 2010 and 2016 were considered in this study.

The inclusion criteria were as follows: (1) SCLC as the only histologically confirmed primary tumor, (2) patients with $\mathrm{BM}$, and (3) patients with complete clinicopathologic features, demographic data, and survival information. Finally, we extracted 6253 SCLC patients with BM at initial diagnosis from 309,056 lung cancer patients. The study population was randomly divided into training and validation cohorts at a $7: 3$ ratio, and the classification process was performed using $\mathrm{R}$ software.

2.2. Ethics Statement. This study was based on publicly available data from the SEER database (https://seer.cancer. gov/), and a data use agreement was signed. The SEER database does not include personally identifiable information, and because no direct interaction with patients occurred in this study, ethics exemption was obtained from the ethics committee of the local hospital for this study.

2.3. Variable Definitions. Based on patient-specific information in the SEER database, we selected 16 variables to identify independent prognostic factors for SCLC with BM, including age, sex, race, primary site, grade, laterality, $T$ stage, $N$ stage, distant metastatic sites (lung, brain, and liver), surgery, radiotherapy, chemotherapy, insurance status, and marital status. The primary tumor site is defined according to the International Classification of Diseases for Oncology (ICD-O) code: upper lobe of the lung (C34.1), middle lobe of the lung (C34.2), lower lobe of the lung (C34.3), and lung, if not otherwise specified (C34.9). Third edition (ICD-O-3) histology codes, as follows, were used to identify cases with SCLC: 8002 (malignant tumor, small cell type), 8041 (small cell carcinoma, NOS), 8042 (oat cell carcinoma), 8043 (small cell carcinoma, fusiform cell), 8044 (small cell carcinoma, intermediate cell), and 8045 (combined small cell carcinoma). All cases in this study were classified according to the $7^{\text {th }}$ edition of the American Joint Committee on Cancer TNM staging system as grade I (well-differentiated), grade II (moderately differentiated), grade III (poorly differentiated), or grade IV (undifferentiated). Distant organ metastasis is defined by the SEER program as the state of metastasis in distant organs at the time of the first diagnosis of cancer, where the sites of metastasis recorded include the bone, liver, brain, and lung. Regarding marital status, we excluded misleading data on unmarried or domestic partners and then included "unmarried," "separated," "single," and "widowed" all in the unmarried group. Insurance status is divided into insured and uninsured, with both "insured" and "insured/unspecific" included in the insured group. In the survival analysis, the primary endpoint of our study was overall survival (OS), which was defined as the date from diagnosis to death (from any cause) or the date of the last follow-up.

2.4. Statistical Analysis. The chi-square test was used for categorical data. The optimal cutoff value of age in terms of OS was determined by $X$-tile software (Yale University, New Haven, CT, USA). To process the data conveniently, we divided the patients into three groups according to age $(<66$, $67-79$, and $>79$ years) [17]. Univariate and multivariate Cox proportional hazards regression analyses of the training cohort were used to identify independent prognostic factors from which predictive models were constructed. Receiver operating characteristic (ROC) curves and the area under the curve (AUC) were used to evaluate the discrimination of the nomogram. The calibration curve is a graphical display of calibration accuracy and is used to measure the agreement of predicted probabilities with actual survival outcomes. To further assess the benefits and advantages of the predictive model, we used decision curve analysis (DCA). All evaluation processes were conducted 1000 times using bootstrapping. Finally, all patients were divided into high-risk and low-risk groups according to the median risk score, and 


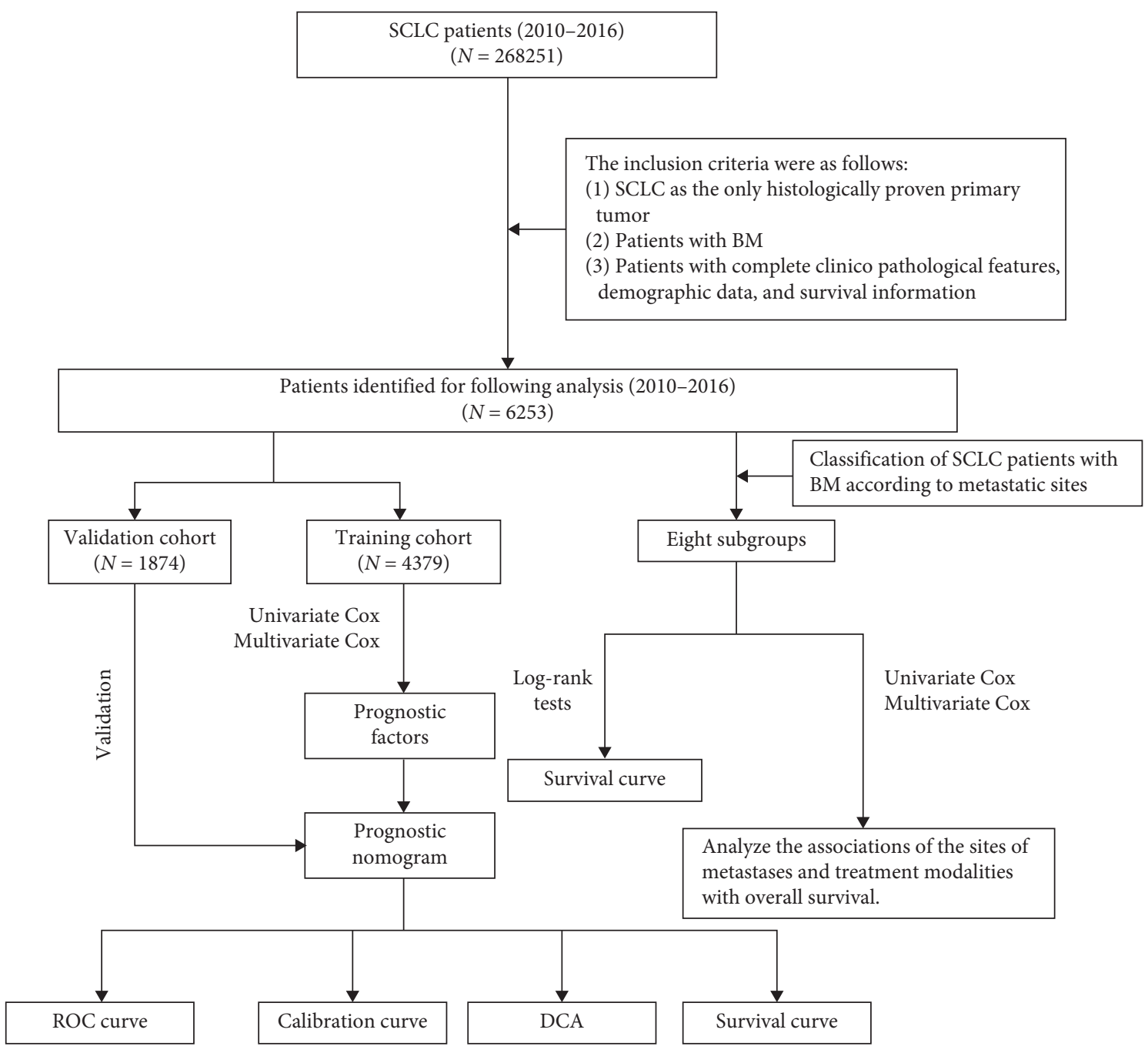

FIgURE 1: The workflow describing the schematic overview of the project.

survival curves were used to verify the prognostic value of the nomogram [18].

Patients with SCLC were classified according to the site of metastasis. A Kaplan-Meier analysis was used to assess survival time for each subgroup of patients, and differences in survival time were determined using the log-rank test. A Cox proportional hazards regression analysis was used to analyze the relationship among metastasis sites, treatment modality, and OS. This study used SPSS 25.0 (NY, USA) and $\mathrm{R}$ software (version 3.6.1) for the statistical analysis. In the present study, a $p$ value $<0.05$ (two-sided) indicated statistical significance.

\section{Results}

3.1. Baseline Characteristics of the Study Population. Ultimately, 6253 SCLC patients with BM at initial diagnosis were identified from the SEER database and were randomized at a $7: 3$ ratio into a training cohort $(n=4379)$ and a validation cohort $(n=1874)$. Table 1 summarizes the demographic and clinical characteristics of the SCLC patients with BM. Of all the patients included, 2941 (47.03\%) were aged 66-79 years, and the majority of patients were male (55.56\%) and white (88.97\%). A total of $1408(22.52 \%)$ patients had lung metastases, 1190 (19.03\%) had brain metastases, and $3530(56.45 \%)$ had liver metastases. The most common $T$ and $N$ stages were $T 4(30.90 \%)$ and $N 2$ (47.37\%). Regarding therapy, $138(2.21 \%)$ of the patients underwent surgery, 4499 (71.95\%) received chemotherapy, and 2417 (38.65\%) received radiotherapy.

3.2. Prognostic Factors for SCLC Patients with BM. Univariate and multivariate Cox proportional hazards regression analyses were performed to screen for prognostic factors. The results of the Cox proportional hazards regression analysis performed for all patients are given in Table 2. In the univariate Cox regression analysis, age, sex, primary site, $T$ stage, radiotherapy, chemotherapy, brain metastases, liver metastases, lung metastases, insurance status, and marital status were significantly associated with OS. Finally, the results of the multivariate Cox regression analysis showed that age, sex, primary site, radiotherapy, chemotherapy, brain metastases, liver metastases, and 
TABLE 1: Demographic and clinical characteristics of SCLC patients with BM.

\begin{tabular}{|c|c|c|c|c|c|c|}
\hline \multirow[t]{2}{*}{ Variables } & \multicolumn{2}{|c|}{$\begin{array}{c}\text { Total cohort } \\
N=6253\end{array}$} & \multicolumn{2}{|c|}{$\begin{array}{l}\text { Training cohort } \\
\qquad N=4379\end{array}$} & \multicolumn{2}{|c|}{$\begin{array}{l}\text { Validation cohort } \\
\qquad N=1874\end{array}$} \\
\hline & $n$ & $\%$ & $n$ & $\%$ & $n$ & $\%$ \\
\hline \multicolumn{7}{|l|}{$\overline{\text { Age }}$} \\
\hline$<66$ & 2647 & 42.33 & 1824 & 41.65 & 823 & 43.92 \\
\hline $66-79$ & 2941 & 47.03 & 2082 & 47.55 & 859 & 45.84 \\
\hline$>79$ & 665 & 10.63 & 473 & 10.80 & 192 & 10.25 \\
\hline \multicolumn{7}{|l|}{ Race } \\
\hline Black & 419 & 6.70 & 292 & 6.67 & 127 & 6.78 \\
\hline Others & 267 & 4.27 & 191 & 4.36 & 76 & 4.06 \\
\hline White & 5563 & 88.97 & 3894 & 88.92 & 1669 & 89.06 \\
\hline Unknown & 4 & 0.06 & 2 & 0.05 & 2 & 0.11 \\
\hline \multicolumn{7}{|l|}{ Sex } \\
\hline Female & 2779 & 44.44 & 1948 & 44.49 & 831 & 44.34 \\
\hline Male & 3474 & 55.56 & 2431 & 55.51 & 1043 & 55.66 \\
\hline \multicolumn{7}{|l|}{ Primary site } \\
\hline Upper lobe & 2857 & 45.69 & 2018 & 46.08 & 839 & 44.77 \\
\hline Middle lobe & 219 & 3.50 & 158 & 3.61 & 61 & 3.26 \\
\hline Lower lobe & 1260 & 20.15 & 865 & 19.75 & 395 & 21.08 \\
\hline Lung, NOS & 1120 & 17.91 & 770 & 17.58 & 350 & 18.68 \\
\hline Others & 797 & 12.75 & 568 & 12.97 & 229 & 12.22 \\
\hline \multicolumn{7}{|l|}{ Grade } \\
\hline I & 7 & 0.11 & 6 & 0.14 & 1 & 0.05 \\
\hline II & 8 & 0.13 & 6 & 0.14 & 2 & 0.11 \\
\hline III & 457 & 7.31 & 317 & 7.24 & 140 & 7.47 \\
\hline IV & 757 & 12.11 & 526 & 12.01 & 231 & 12.33 \\
\hline Unknown & 5024 & 80.35 & 3524 & 80.48 & 1500 & 80.04 \\
\hline \multicolumn{7}{|l|}{ Laterality } \\
\hline Bilateral & 89 & 1.42 & 58 & 1.32 & 31 & 1.65 \\
\hline Left & 2519 & 40.28 & 1771 & 40.44 & 748 & 39.91 \\
\hline Right & 3286 & 52.55 & 2297 & 52.45 & 989 & 52.77 \\
\hline Unknown & 359 & 5.74 & 253 & 5.78 & 106 & 5.66 \\
\hline \multicolumn{7}{|l|}{$T$ stage } \\
\hline$T 1$ & 434 & 6.94 & 292 & 6.67 & 142 & 7.58 \\
\hline$T 2$ & 1145 & 18.31 & 801 & 18.29 & 344 & 18.36 \\
\hline T3 & 1094 & 17.50 & 744 & 16.99 & 350 & 18.68 \\
\hline$T 4$ & 1932 & 30.90 & 1390 & 31.74 & 542 & 28.92 \\
\hline Unknown & 1648 & 26.36 & 1152 & 26.31 & 496 & 26.47 \\
\hline \multicolumn{7}{|l|}{$N$ stage } \\
\hline NO & 499 & 7.98 & 344 & 7.86 & 155 & 8.27 \\
\hline$N 1$ & 306 & 4.89 & 227 & 5.18 & 79 & 4.22 \\
\hline N2 & 2962 & 47.37 & 2100 & 47.96 & 862 & 46.00 \\
\hline$N 3$ & 1455 & 23.27 & 992 & 22.65 & 463 & 24.71 \\
\hline Unknown & 1031 & 16.49 & 716 & 16.35 & 315 & 16.81 \\
\hline \multicolumn{7}{|l|}{ Radiotherapy } \\
\hline No & 3836 & 61.35 & 2696 & 61.57 & 1140 & 60.83 \\
\hline Yes & 2417 & 38.65 & 1683 & 38.43 & 734 & 39.17 \\
\hline \multicolumn{7}{|l|}{ Chemotherapy } \\
\hline No & 1754 & 28.05 & 1220 & 27.86 & 534 & 28.50 \\
\hline Yes & 4499 & 71.95 & 3159 & 72.14 & 1340 & 71.50 \\
\hline \multicolumn{7}{|l|}{ Surgery } \\
\hline No & 6115 & 97.79 & 4279 & 97.72 & 1836 & 97.97 \\
\hline Yes & 138 & 2.21 & 100 & 2.28 & 38 & 2.03 \\
\hline \multicolumn{7}{|l|}{ Brain metastasis } \\
\hline No & 5063 & 80.97 & 3550 & 81.07 & 1513 & 80.74 \\
\hline Yes & 1190 & 19.03 & 829 & 18.93 & 361 & 19.26 \\
\hline \multicolumn{7}{|l|}{ Liver metastasis } \\
\hline No & 2723 & 43.55 & 1930 & 44.07 & 793 & 42.32 \\
\hline Yes & 3530 & 56.45 & 2449 & 55.93 & 1081 & 57.68 \\
\hline
\end{tabular}


TABle 1: Continued.

\begin{tabular}{|c|c|c|c|c|c|c|}
\hline \multirow{3}{*}{ Variables } & \multicolumn{2}{|c|}{ Total cohort } & \multicolumn{2}{|c|}{ Training cohort } & \multicolumn{2}{|c|}{ Validation cohort } \\
\hline & \multicolumn{2}{|c|}{$N=6253$} & \multicolumn{2}{|c|}{$N=4379$} & \multicolumn{2}{|c|}{$N=1874$} \\
\hline & $n$ & $\%$ & $n$ & $\%$ & $n$ & $\%$ \\
\hline \multicolumn{7}{|c|}{ Lung metastasis } \\
\hline No & 4845 & 77.48 & 3380 & 77.19 & 1465 & 78.18 \\
\hline Yes & 1408 & 22.52 & 999 & 22.81 & 409 & 21.83 \\
\hline \multicolumn{7}{|c|}{ Insurance status } \\
\hline Uninsured & 203 & 3.24 & 140 & 3.20 & 63 & 3.36 \\
\hline Insured & 6050 & 96.75 & 4239 & 96.80 & 1811 & 96.64 \\
\hline \multicolumn{7}{|l|}{ Marital status } \\
\hline Unmarried & 2833 & 45.31 & 1982 & 45.26 & 851 & 45.41 \\
\hline Married & 3420 & 54.69 & 2397 & 54.74 & 1023 & 54.59 \\
\hline
\end{tabular}

SCLC, small cell lung cancer; BM, bone metastasis.

marital status were independent prognostic factors (Table 2). Patients who received radiotherapy and chemotherapy had a lower risk of death with a distribution hazard ratio of 0.801 (95\% CI: $0.747-0.860$ ) and 0.272 (95\% CI: 0.252-0.294), respectively. In addition, advanced age, male sex, unknown primary site, liver and brain metastases, and unmarried status were associated with a higher risk of death.

\subsection{Prognostic Nomogram Development and Validation.} Based on the prognostic factors selected in the training cohort, a nomogram was established to predict the OS of SCLC patients with BM (Figure 2). In the prognostic nomogram, values for the individual patient are located along the variable axes, and a line is drawn upward to the points' axis to determine the number of points assigned for each variable. The scores for each variable are then summed to calculate an individual's total risk score, and the 6-, 12-, and 18 -month OS are estimated visually by drawing a line from the total score axis to the 6-, 12-, and 18-month survival probability axes. We plotted the ROC curves for the training and validation cohorts and calculated the corresponding AUCs. The AUCs of the nomogram for the 6-, 12-, and 18month OS reached $0.776,0.739$, and 0.752 , respectively, in the training cohort and $0.787,0.750$, and 0.743 in the validation cohort, respectively (Figure 3 ). In addition, we further compared the difference in the AUC value between the nomogram and all independent prognostic factors, and the results showed that the AUC value of the nomogram was higher than the AUC of all independent factors at 6,12, and 18 months, both in the training cohort and the validation cohort (Figure 3). As shown in Figure 4, calibration curves were generated to verify the agreement between survival, as predicted by the nomogram, and actual observations. These points are close to a 45-degree diagonal, which indicates that we succeeded in achieving the best agreement between the survival rates predicted by the nomogram and the actual survival rates. DCA showed that the prognostic nomogram has a wider and practical range of threshold probabilities, which significantly increases the net benefit and suggests that this nomogram has high clinical utility in predicting OS in SCLC patients with BM (Figure 5).
3.4. Stratification of Risk Groups. Based on the median risk score of patients in the training cohort, all patients, including those in the training and validation cohorts, were divided into low- and high-risk groups. By plotting Kaplan-Meier survival curves, it was easy to observe that patients in the high-risk group exhibited a worse prognosis than those in the low-risk group (Figure 6).

3.5. OS Rates (Median, Mean, 1-, 2-, and 5-Year) of SCLC Patients with BM and Different Sites of Metastasis. Kaplan-Meier survival curve analysis was used to evaluate the prognostic differences among the different metastatic sites. As shown in Table 3 and Figure 7, patients with only BM had the longest mean survival time $(9.30 \pm 0.31$ months $)$, while patients with bone, brain, lung, and liver metastases had the shortest mean survival time ( $4.86 \pm 0.33$ months). In patients with two sites of metastasis, those with bone and liver metastases had a worse prognosis than those with bone and brain metastases and bone and lung metastases. Among the patients with metastases at these three sites, those with bone, lung, and liver metastases had the shortest survival time. We can easily see that as the number of metastatic sites outside the bone increases, patients tend to have a shorter survival time. In addition, if a patient has liver metastases, the survival time is shorter regardless of how many other sites of metastasis are present.

3.6. Relationship among Sites of Metastasis, Treatment Modality, and OS in SCLC Patients with BM. We divided the total cohort into eight subgroups according to the site of metastasis of the patients (bone-only, bone and brain, bone and liver, bone and lung, bone, liver, and brain, bone, lung, and brain, bone, lung, and liver, and bone, lung, liver, and brain). The relationship among the sites of metastasis, treatment modality, and OS is given in Table 4. For all subgroups of patients, chemotherapy was an independent prognostic factor, and all patients who received chemotherapy demonstrated improved OS (all $p<0.001$ ). Radiotherapy positively affected $O S$ in some subgroups (all $p<0.05$ ), which were the bone-only, bone and liver, bone and lung, bone, liver and brain, and bone, liver, brain, and 
TABLE 2: Univariate and multivariate Cox analyses in SCLC patients with BM.

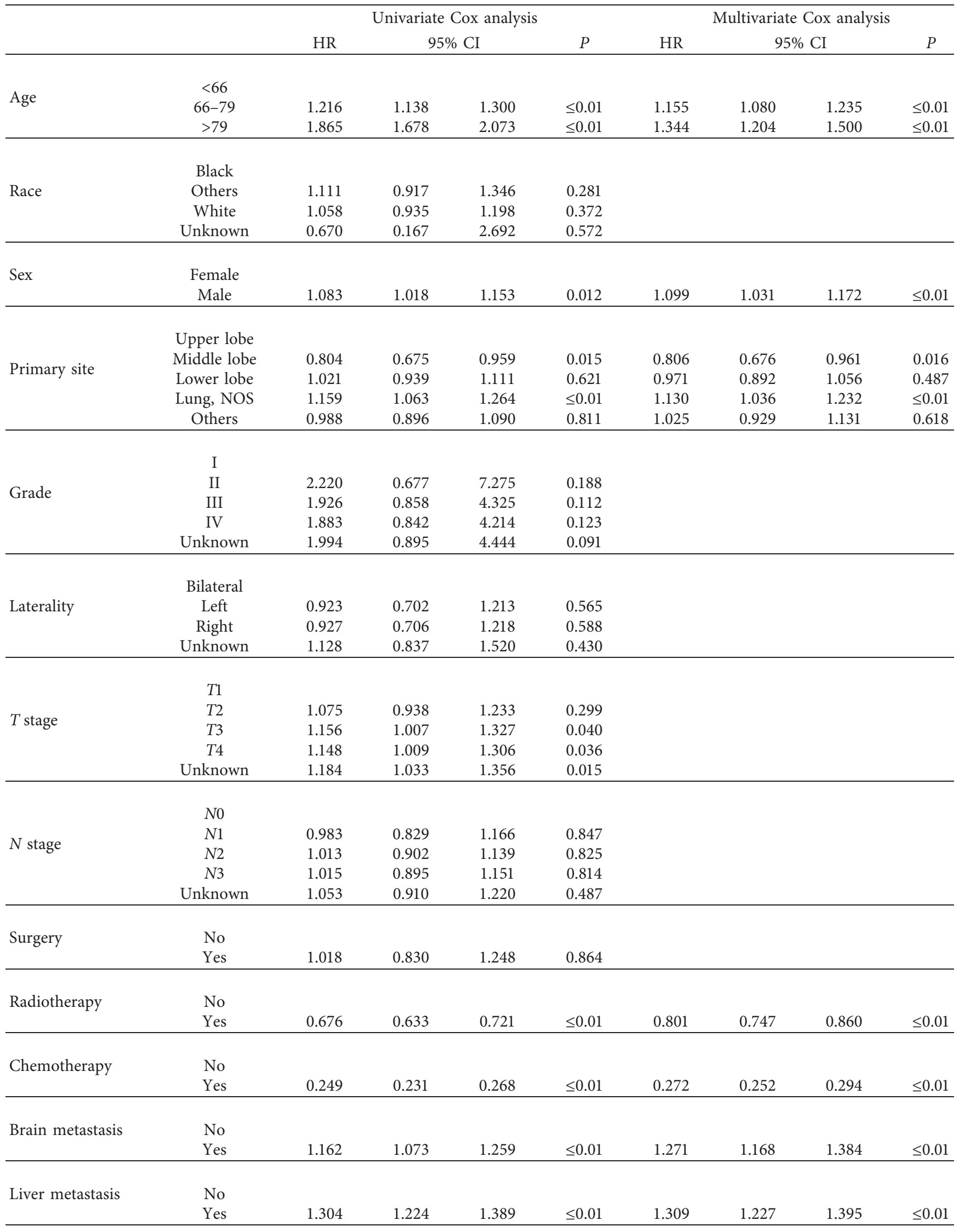


TABLE 2: Continued.

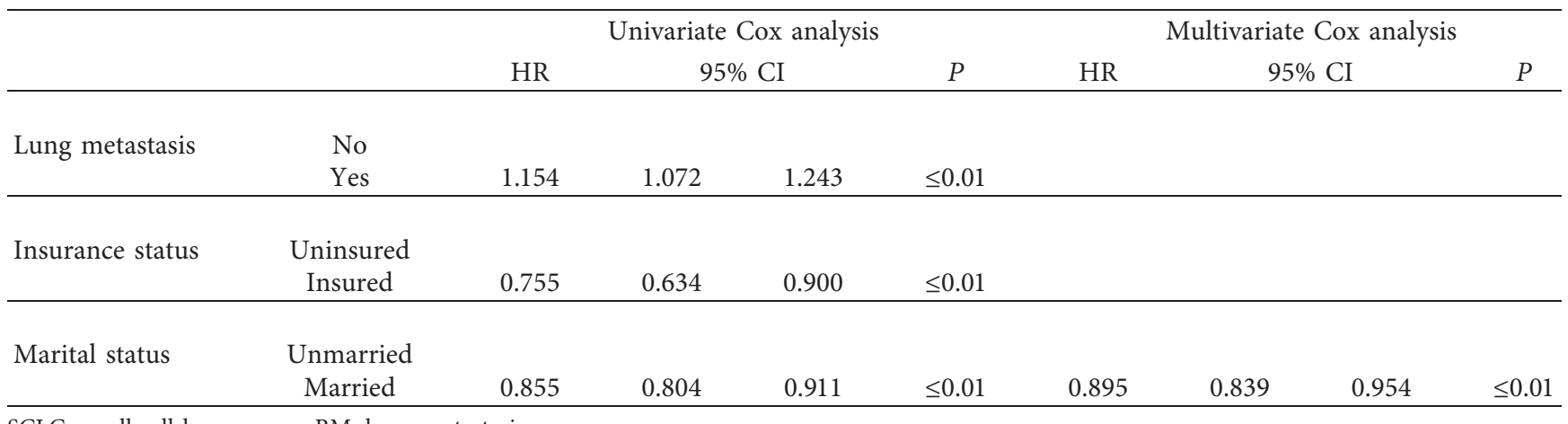

SCLC, small cell lung cancer; BM, bone metastasis.
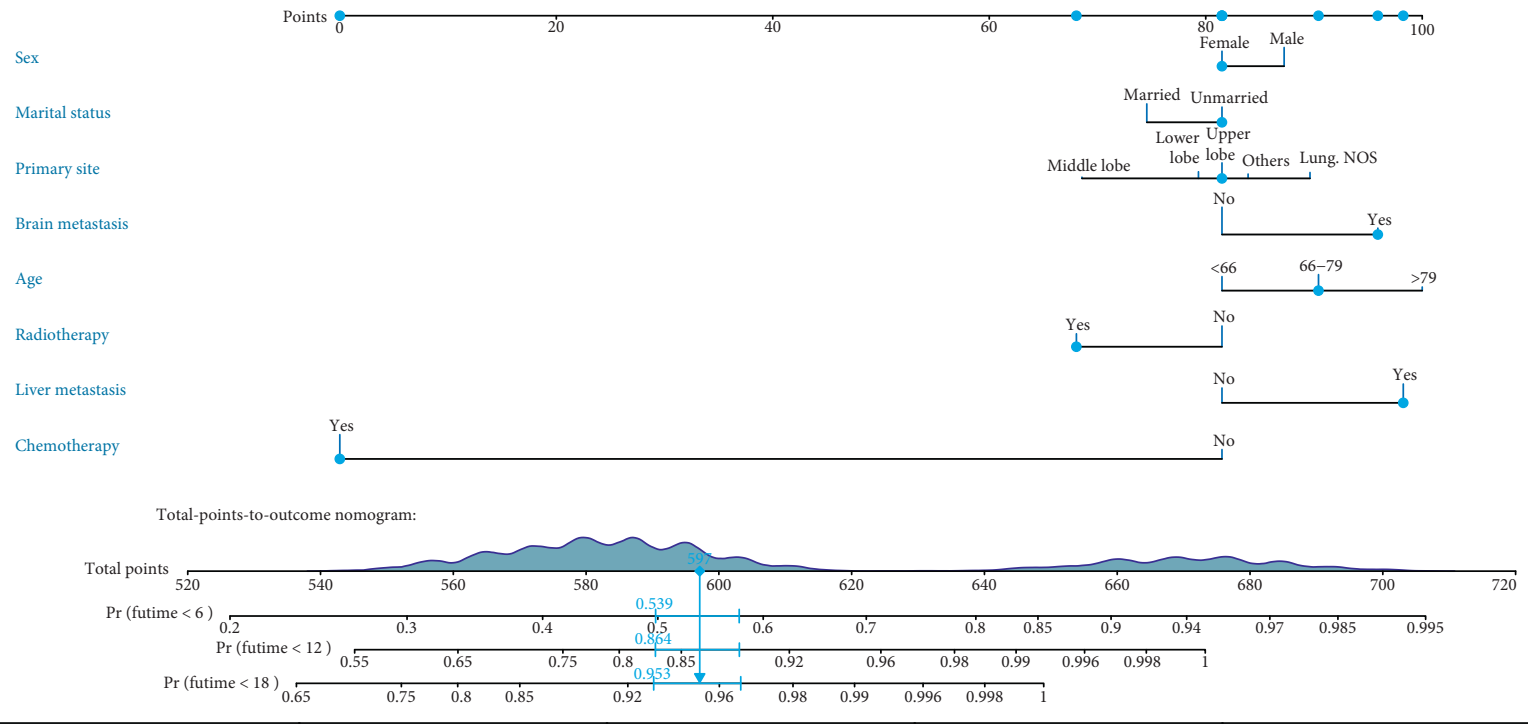

FIgURE 2: A prognostic nomogram for SCLC patients with BM.

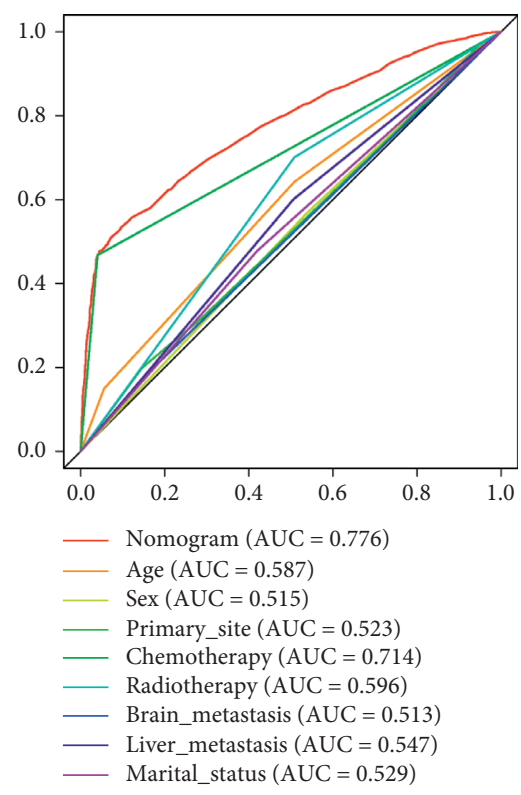

(a)

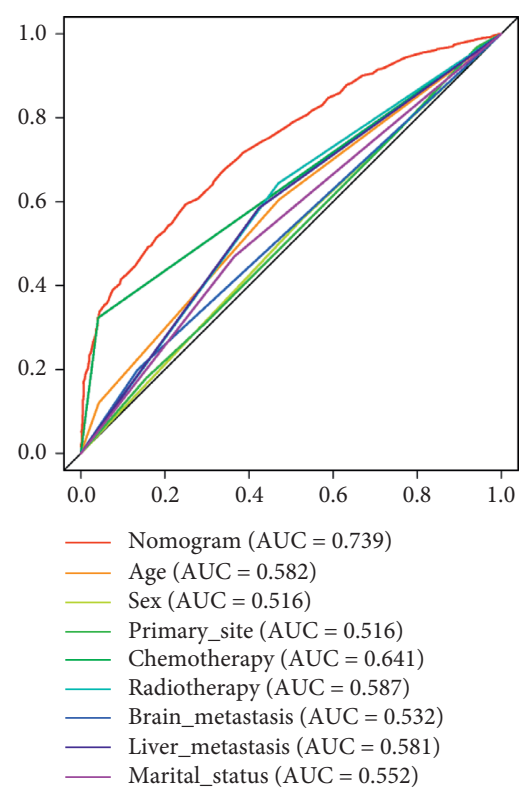

(b)

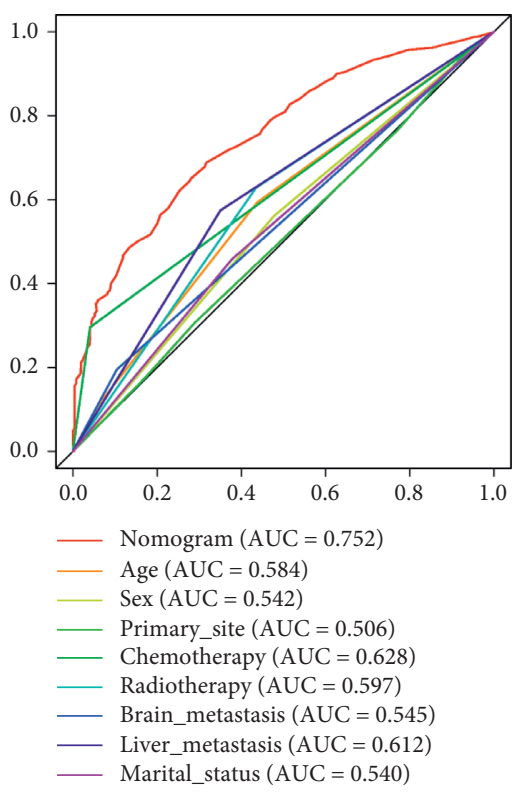

(c)

FIgURE 3: Continued. 


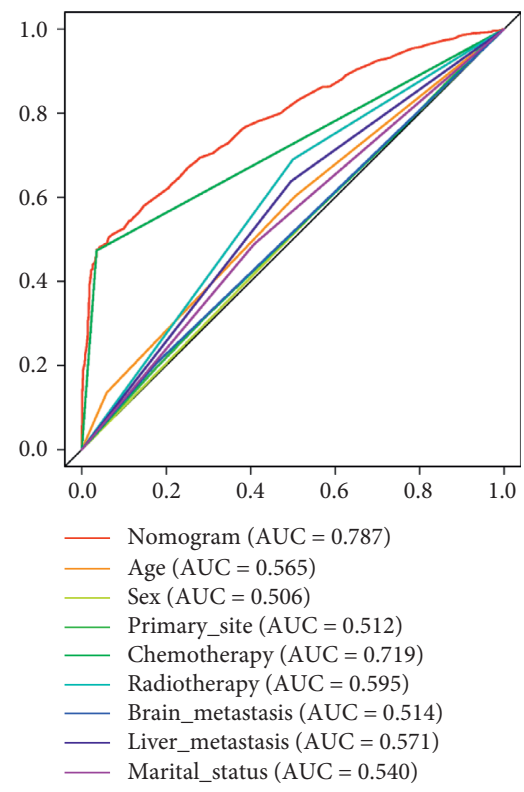

(d)

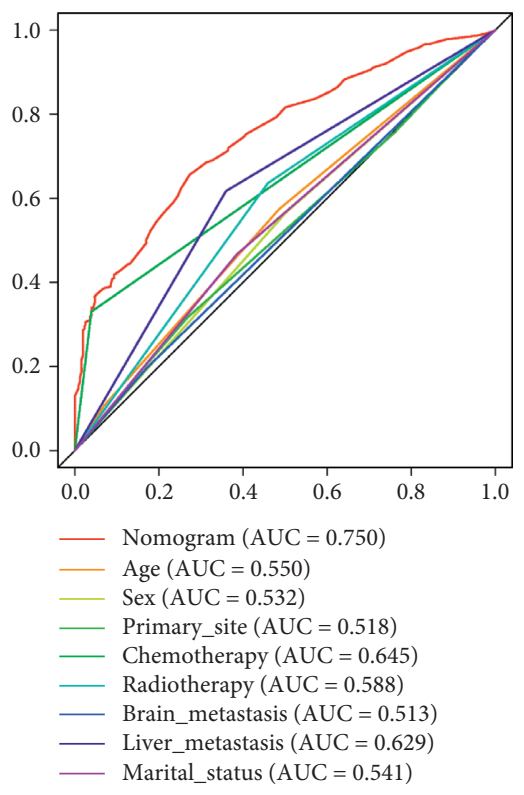

(e)

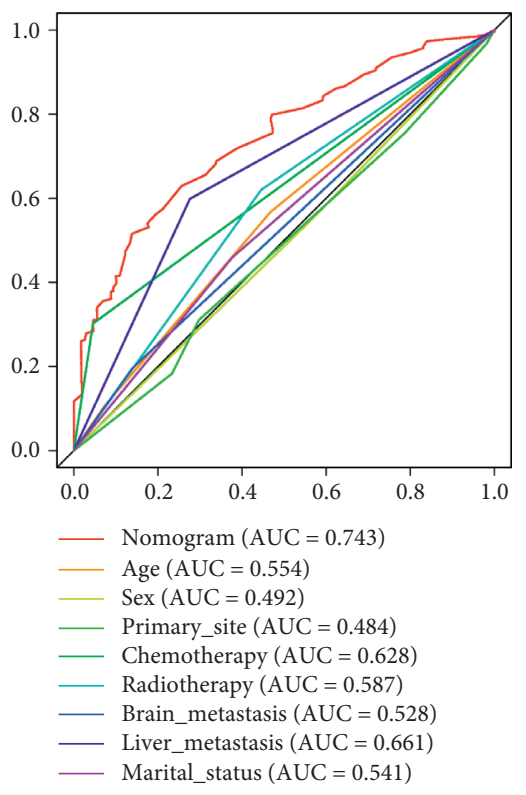

(f)

Figure 3: The ROC curves of the nomogram and all independent predictors at 6 (a), 12 (b), and 18 months (c) in the training cohort and at 6 (d), 12 (e), and 18 months (f) in the validation cohort.

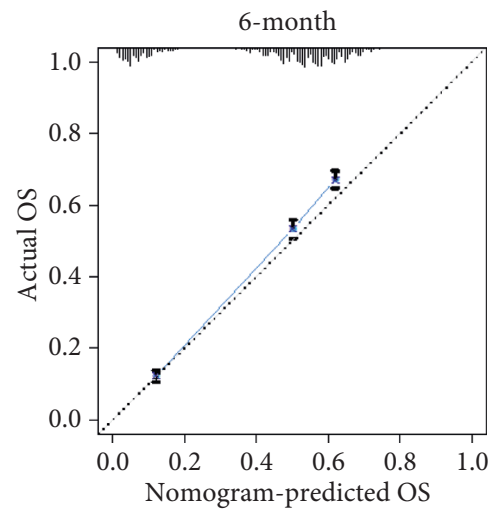

(a)



(d)

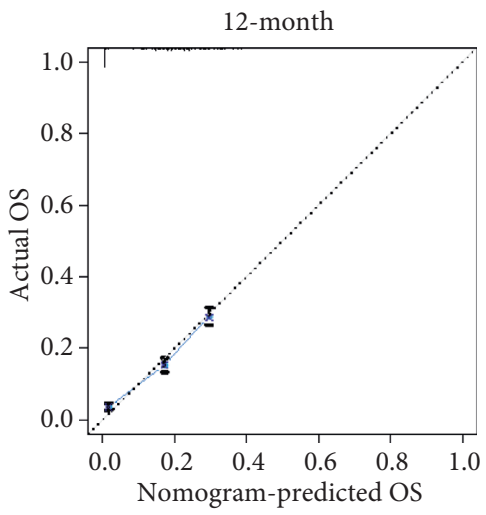

(b)

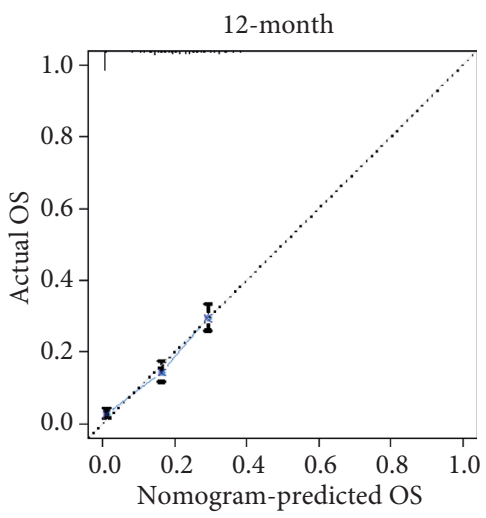

(e)

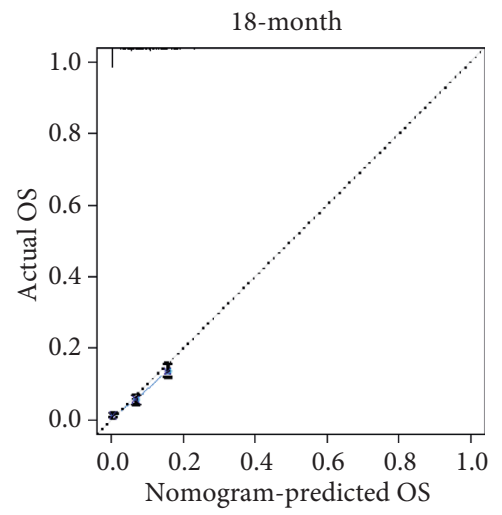

(c)

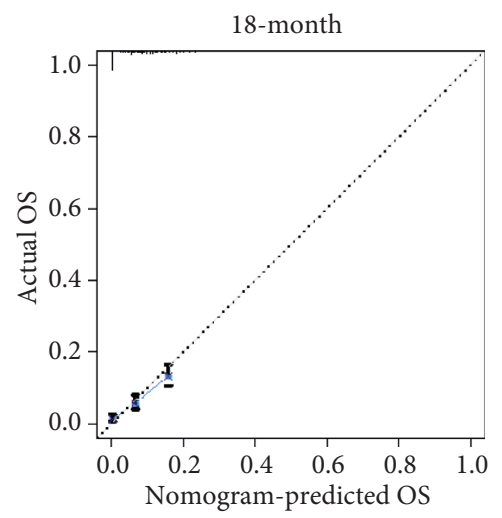

(f)

FIgURE 4: The calibration curves of the nomogram for the prediction of the 6-, 12-, and 18-month OS of patients in the training cohort $((\mathrm{a})-(\mathrm{c}))$ and validation cohort $((\mathrm{d})-(\mathrm{f}))$. The $x$-axis represents the nomogram-predicted survival rates, whereas the $y$-axis represents the actual survival rates. 


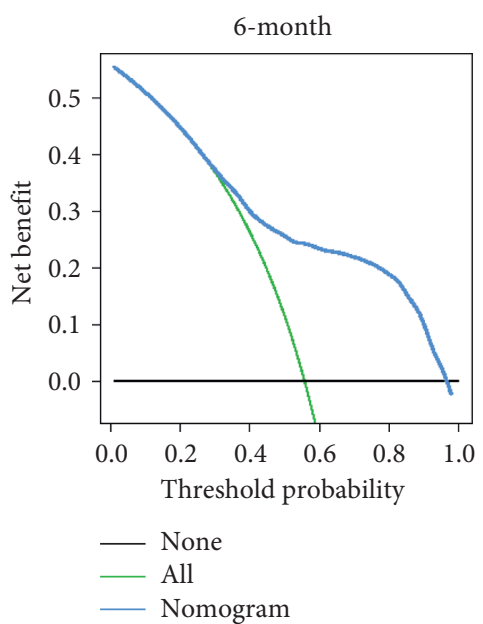

(a)

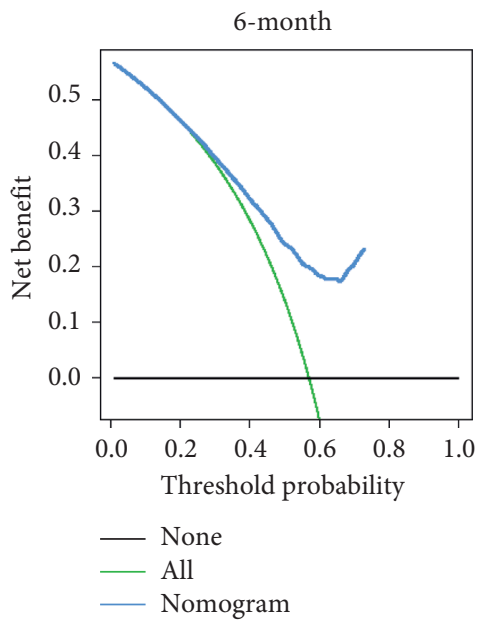

(d)

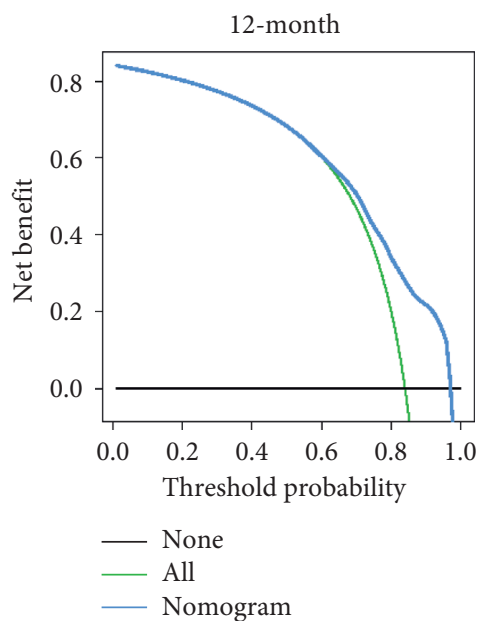

(b)

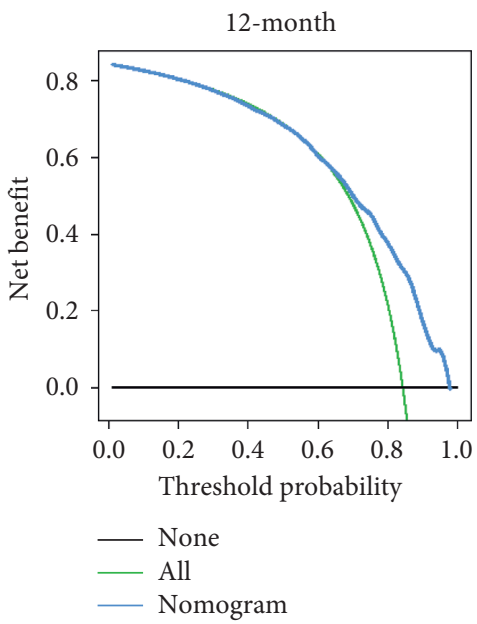

(e)



(c)

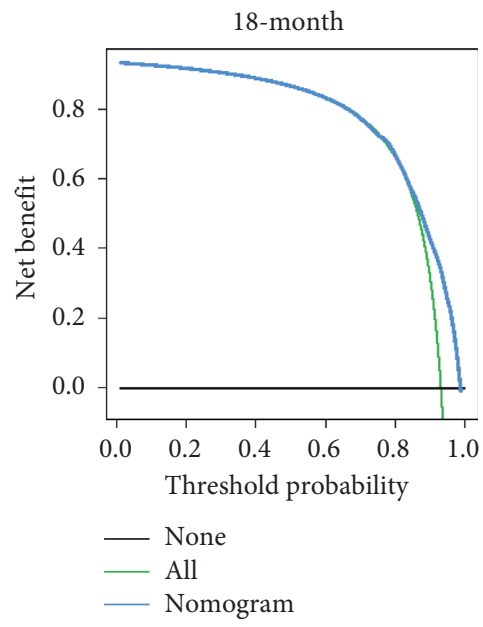

(f)

FIGURE 5: DCA of the nomogram for the survival prediction of SCLC patients with BM. (a) 6-month survival benefit in the training cohort. (b) 12-month survival benefit in the training cohort. (c) 18-month survival benefit in the training cohort. (d) 6-month survival benefit in the validation cohort. (e) 12-month survival benefit in the validation cohort. (f) 18 -month survival benefit in the validation cohort. The $x$-axis shows the threshold probability, and the $y$-axis shows the net benefit rate. The black horizontal line indicates that no patients died. The green oblique line indicates the cancer-specific death for all the patients. The red line represents the prognostic nomogram.

lung metastases subgroups. Surprisingly, surgery was not an independent prognostic factor in any subgroup of patients. For patients in all subgroups, their OS was not affected regardless of whether they underwent surgery (Figure 8), whereas if they received radiotherapy or chemotherapy, their OS was significantly improved (Figures 9 and 10).

\section{Discussion}

In this study, a nomogram model incorporating age, sex, primary site, radiotherapy, chemotherapy, brain metastasis, liver metastasis, and marital status was constructed to predict the probability of OS of patients with SCLC and BM and was validated using an independent validation cohort. The results show that the nomogram model exhibits good discrimination and accuracy in both the training and validation cohorts. In addition, patients were divided into eight subgroups according to the site of metastasis. We further analyzed the prognosis of each subgroup and investigated the survival benefit of the treatment modality (surgery, radiotherapy, and chemotherapy) on each subgroup using a Cox analysis and K-M survival curve analysis.

Few studies have reported the characteristics and prognosis of SCLC patients with BM. To our knowledge, only two studies have reported the prognostic factors in patients with SCLC with BM at initial diagnosis $[13,19]$. However, both the studies by Gong et al. and Kang et al. have significant shortcomings. First, the results are not convincing due to the small sample sizes of 102 and 61 cases, whereas our study included 6253 patients from the SEER database. Since the SEER database is one of the largest open cancer databases globally and covers approximately $28 \%$ of the United States population, the results of this study are not the only representative but also highly reliable. Second, some 


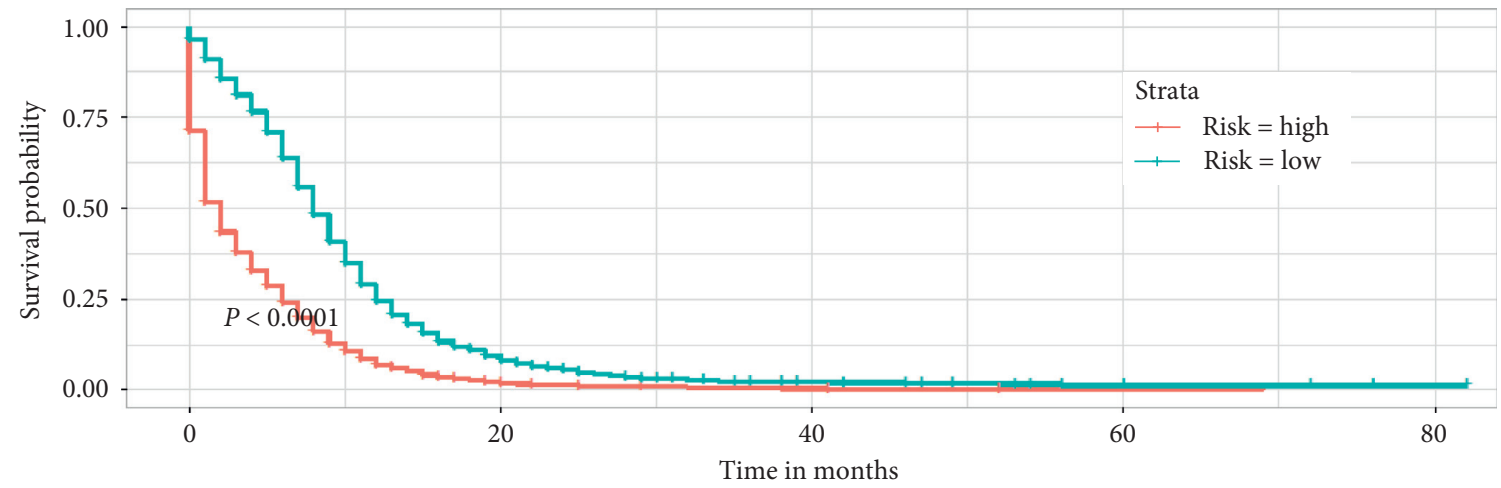

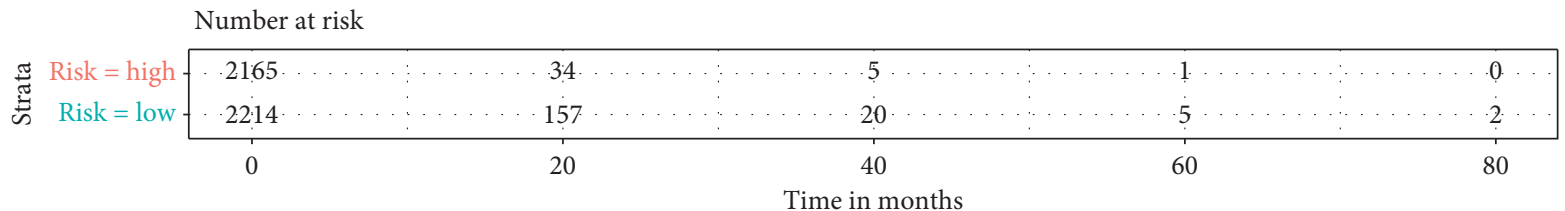

(a)

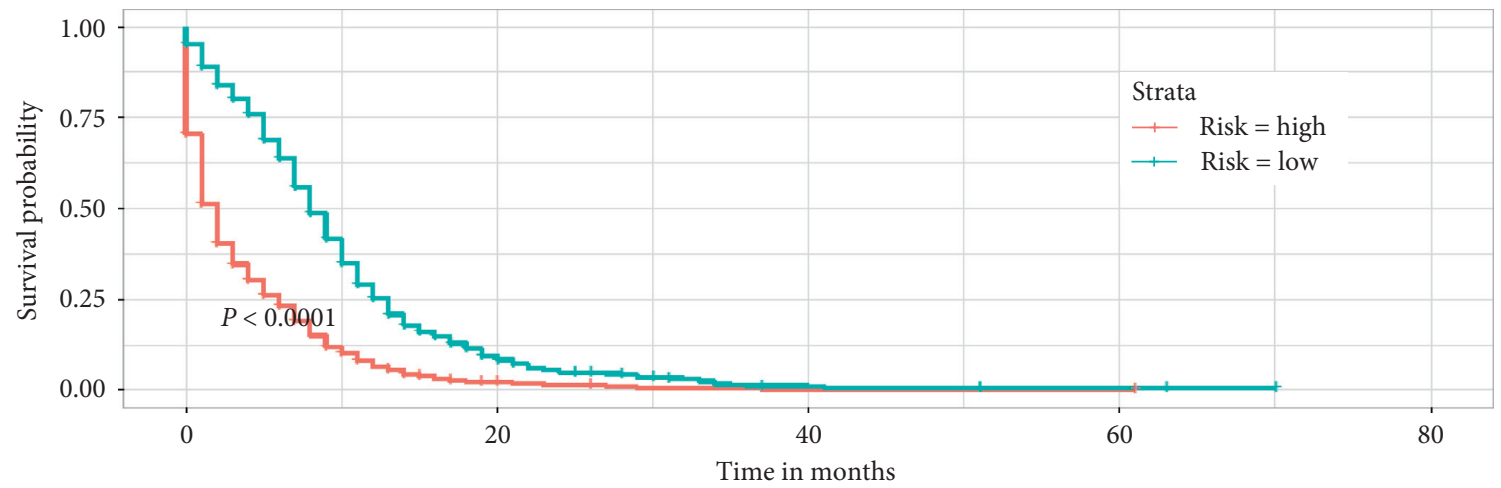

Number at risk

\begin{tabular}{|c|c|c|c|c|}
\hline \multirow{3}{*}{ 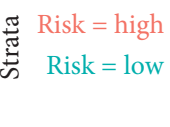 } & . 966 & 13 & 1 & \\
\hline & 908 & 64 & 5 & 2 \\
\hline & 0 & 20 & 40 & 6 \\
\hline
\end{tabular}

(b)

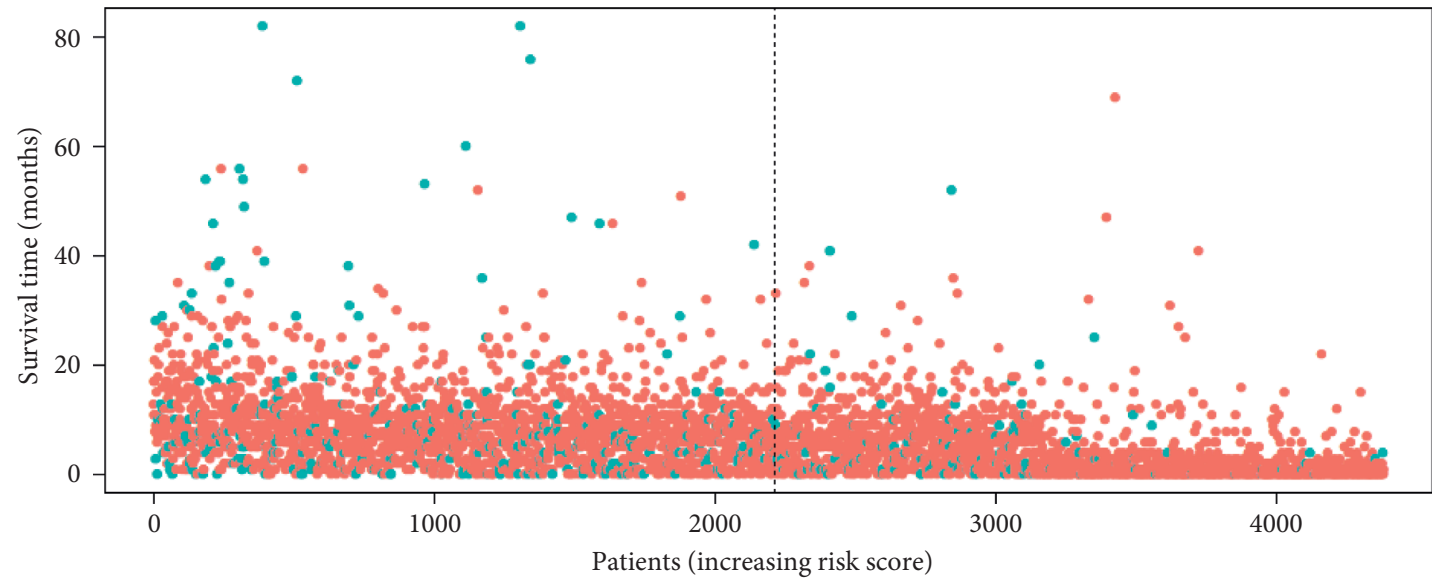

(c)

FIgURE 6: Continued. 


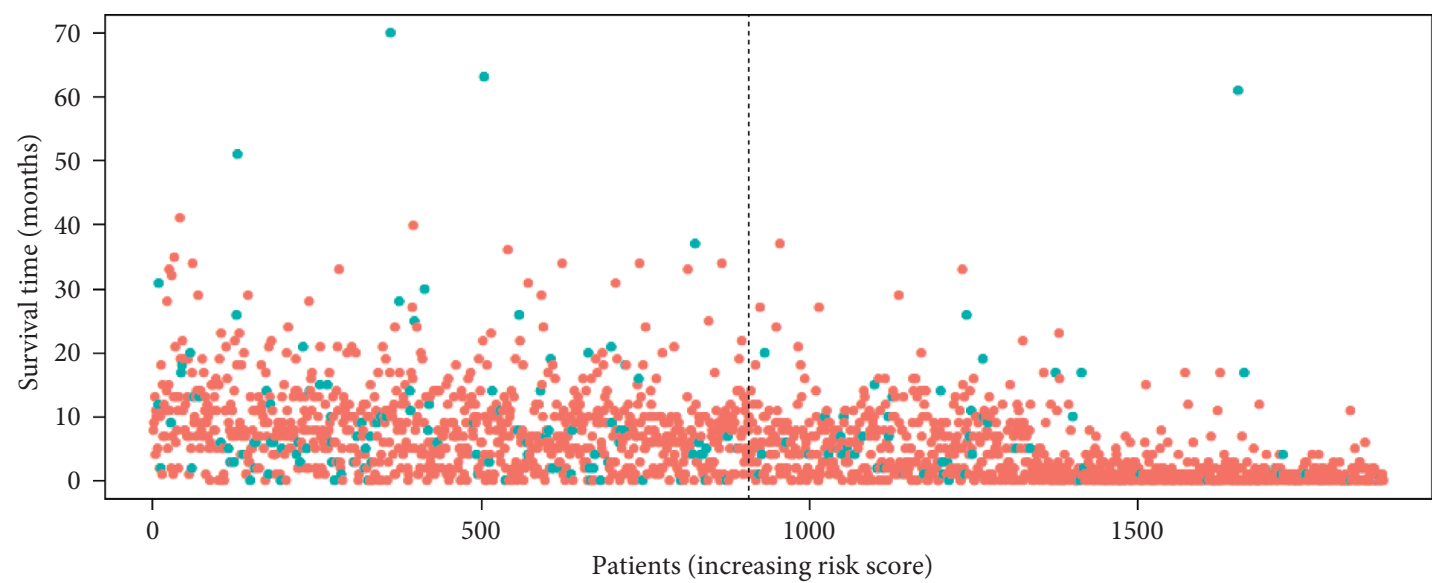

(d)

Figure 6: Kaplan-Meier survival analysis of the signature for both the training cohort and the validation cohort. Patients in the training cohort ((a), (c)) and validation cohort ((b), (d)) with a higher risk score demonstrated a worse OS than those with a lower risk score, which suggests the strong predictive ability of the nomogram for the survival outcomes of patients with BM.

TABLE 3: OS rates (median, mean) of SCLC patients with BM in different metastatic sites.

\begin{tabular}{lcc}
\hline Metastatic sites & Median survival (months) & Mean survival (months) \\
\hline Bone-only & $7.00 \pm 0.25$ & $9.30 \pm 0.31$ \\
Bone and brain & $5.00 \pm 0.52$ & $7.12 \pm 0.40$ \\
Bone and liver & $5.00 \pm 0.20$ & $6.45 \pm 0.20$ \\
Bone and lung & $6.00 \pm 0.53$ & $7.63 \pm 0.49$ \\
Bone, liver, and brain & $5.00 \pm 0.45$ & $5.77 \pm 0.29$ \\
Bone, lung, and brain & $5.00 \pm 1.04$ & $6.69 \pm 0.74$ \\
Bone, lung, and liver & $4.00 \pm 0.39$ & $5.34 \pm 0.24$ \\
Bone, lung, liver, and brain & $3.00 \pm 0.44$ & $4.86 \pm 0.33$ \\
\hline
\end{tabular}

OS, overall survival; SCLC, small cell lung cancer; BM, bone metastasis.
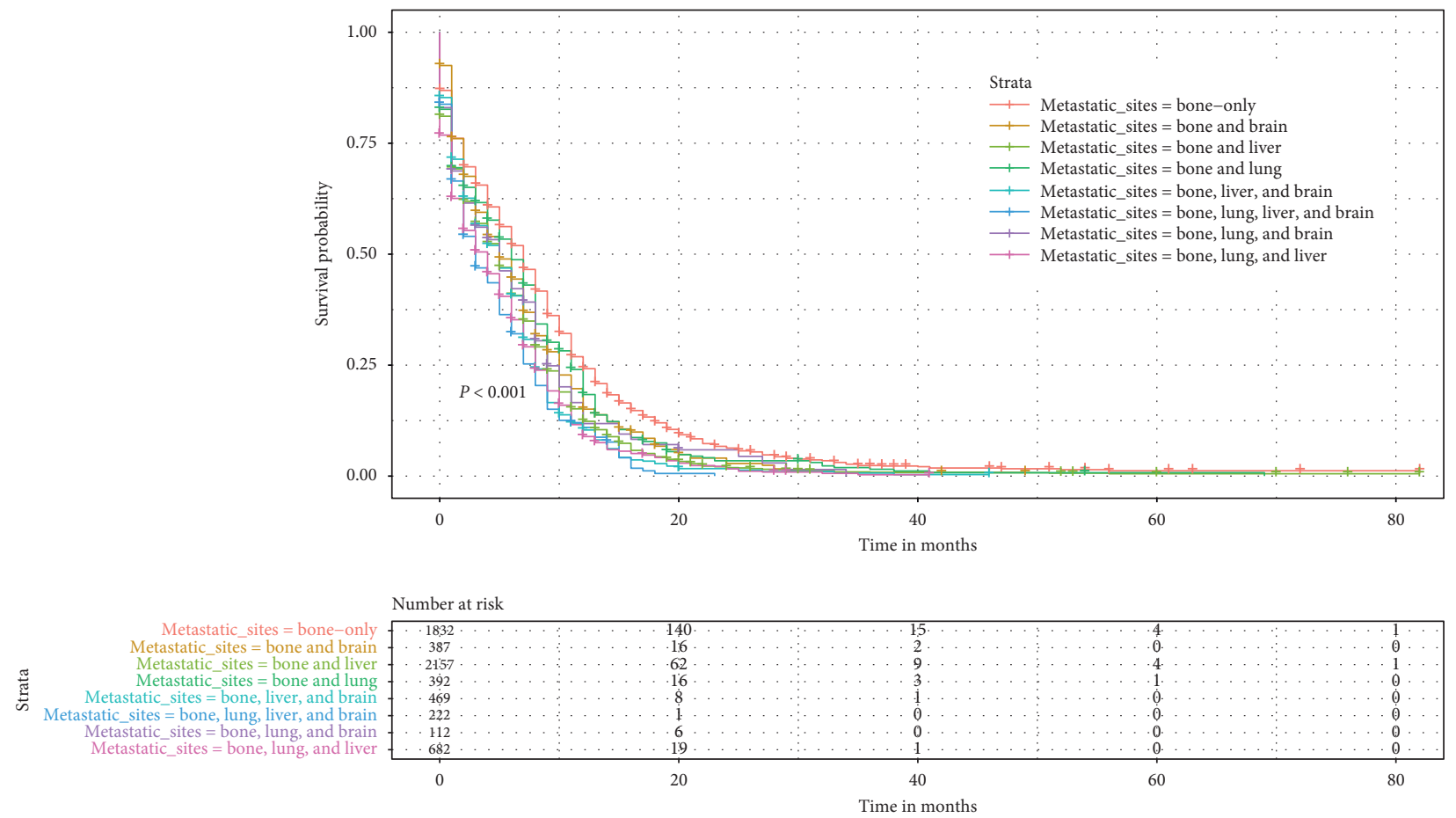

FIGURE 7: Kaplan-Meier survival curves and log-rank tests showed statistically significant differences in the OS of SCLC patients with BM and different metastatic sites. 
TABLE 4: Univariate and multivariate Cox regression for overall survival based on treatment modalities and metastatic sites.

\begin{tabular}{|c|c|c|c|c|c|c|c|}
\hline Groups $(n)$ & Variables & Level & $n$ & $\begin{array}{c}\text { Univariate } \\
\text { HR (95\% CI) }\end{array}$ & $P$ value & $\begin{array}{c}\text { Multivariate } \\
\text { HR (95\% CI) }\end{array}$ & $P$ value \\
\hline \multirow{6}{*}{ Bone-only (1832) } & \multirow{2}{*}{ Surgery } & No & 1786 & & & & \\
\hline & & Yes & 46 & $1.123(0.829-1.520)$ & 0.454 & & \\
\hline & \multirow{2}{*}{ Radiotherapy } & No & 1117 & & & & \\
\hline & & Yes & 715 & $0.613(0.554-0.679)$ & $\leq 0.01$ & $0.784(0.705-0.873)$ & $\leq 0.01$ \\
\hline & \multirow{2}{*}{ Chemotherapy } & No & 485 & & & & \\
\hline & & Yes & 1347 & $0.239(0.212-0.268)$ & $\leq 0.01$ & $0.262(0.231-0.297)$ & $\leq 0.01$ \\
\hline \multirow{6}{*}{ Bone and brain (387) } & \multirow{2}{*}{ Surgery } & No & 377 & & & & \\
\hline & & Yes & 10 & $1.471(0.758-2.856)$ & 0.254 & & \\
\hline & \multirow{2}{*}{ Radiotherapy } & No & 94 & & & & \\
\hline & & Yes & 293 & $0.698(0.546-0.893)$ & $\leq 0.01$ & & \\
\hline & \multirow{2}{*}{ Chemotherapy } & No & 97 & & & & \\
\hline & & Yes & 290 & $0.233(0.178-0.304)$ & $\leq 0.01$ & $0.233(0.178-0.304)$ & $\leq 0.01$ \\
\hline \multirow{6}{*}{ Bone and liver (2157) } & \multirow{2}{*}{ Surgery } & No & 2118 & & & & \\
\hline & & Yes & 39 & $0.992(0.713-1.380)$ & 0.962 & & \\
\hline & \multirow{2}{*}{ Radiotherapy } & No & 1553 & & & & \\
\hline & & Yes & 604 & $0.675(0.611-0.745)$ & $\leq 0.01$ & $0.794(0.717-0.878)$ & $\leq 0.01$ \\
\hline & \multirow{2}{*}{ Chemotherapy } & No & 608 & & & & \\
\hline & & Yes & 1549 & $0.229(0.205-0.254)$ & $\leq 0.01$ & $0.243(0.218-0.271)$ & $\leq 0.01$ \\
\hline \multirow{6}{*}{ Bone and lung (392) } & \multirow{2}{*}{ Surgery } & No & 383 & & & & \\
\hline & & Yes & 9 & $0.822(0.420-1.610)$ & 0.567 & & \\
\hline & \multirow{2}{*}{ Radiotherapy } & No & 265 & & & & \\
\hline & & Yes & 127 & $0.654(0.523-0.817)$ & $\leq 0.01$ & $0.777(0.618-0.976)$ & 0.030 \\
\hline & \multirow{2}{*}{ Chemotherapy } & No & 119 & & & & \\
\hline & & Yes & 273 & $0.345(0.273-0.436)$ & $\leq 0.01$ & $0.367(0.289-0.467)$ & $\leq 0.01$ \\
\hline \multirow{6}{*}{ Bone, liver, and brain (469) } & \multirow{2}{*}{ Surgery } & No & 460 & & & & \\
\hline & & Yes & 9 & $0.919(0.475-1.781)$ & 0.803 & & \\
\hline & \multirow{2}{*}{ Radiotherapy } & No & 165 & & & & \\
\hline & & Yes & 304 & $0.616(0.504-0.752)$ & $\leq 0.01$ & $0.785(0.637-0.968)$ & 0.024 \\
\hline & \multirow{2}{*}{ Chemotherapy } & No & 130 & & & & \\
\hline & & Yes & 339 & $0.247(0.195-0.311)$ & $\leq 0.01$ & $0.264(0.208-0.336)$ & $\leq 0.01$ \\
\hline \multirow{6}{*}{ Bone, lung, and brain (112) } & & No & 108 & & & & \\
\hline & Surgery & Yes & 4 & $1.402(0.513-3.830)$ & 0.510 & & \\
\hline & & No & 34 & & & & \\
\hline & Radiotherapy & Yes & 78 & $0.557(0.361-0.859)$ & $\leq 0.01$ & & \\
\hline & & No & 32 & & & & \\
\hline & Chemotherapy & Yes & 80 & $0.265(0.168-0.419)$ & $\leq 0.01$ & $0.210(0.127-0.348)$ & $\leq 0.01$ \\
\hline & & No & 666 & & & & \\
\hline & Surgery & Yes & 16 & $0.952(0.576-1.573)$ & 0.848 & & \\
\hline Bone lung and liver (682) & Radiotherany & No & 522 & & & & \\
\hline Bone, lung, and liver (682) & Radiotherapy & Yes & 160 & $0.751(0.626-0.903)$ & $\leq 0.01$ & & \\
\hline & & No & 224 & & & & \\
\hline & Chemotherapy & Yes & 458 & $0.259(0.216-0.311)$ & $\leq 0.01$ & $0.262(0.218-0.314)$ & $\leq 0.01$ \\
\hline & & No & 217 & & & & \\
\hline & Surgery & Yes & 5 & $1.145(0.470-2.786)$ & 0.766 & & \\
\hline Bone, lung liver and brain (222) & Radiotherany & No & 86 & & & & \\
\hline Bone, rung, ilver, and oram (LLL) & Kadotnerapy & Yes & 136 & $0.594(0.447-0.789)$ & $\leq 0.01$ & $0.681(0.508-0.914)$ & 0.011 \\
\hline & & No & 59 & & & & \\
\hline & Chemotherapy & Yes & 163 & $0.246(0.175-0.345)$ & $\leq 0.01$ & $0.261(0.183-0.373)$ & $\leq 0.01$ \\
\hline
\end{tabular}

important tumor characteristics and demographic characteristics, such as grade, primary tumor site, laterality, marital status, and insurance status, were not included as variables in the other two studies. Instead, we not only included as many potentially relevant variables as possible but we also constructed a nomogram model based on independent prognostic factors that accurately predicted the prognosis of SCLC patients with BM at initial diagnosis. Compared with the scoring system reported by Gong et al., the nomogram model we constructed has the advantage of visualization of each variable score and individualized survival probability, as well as a more excellent clinical utility [13].

In previous studies, advanced age has been reported to be a poor prognostic factor in patients with SCLC [20-22]. Our study shows that older SCLC patients with BM at initial diagnosis have a higher risk of death. First, this 


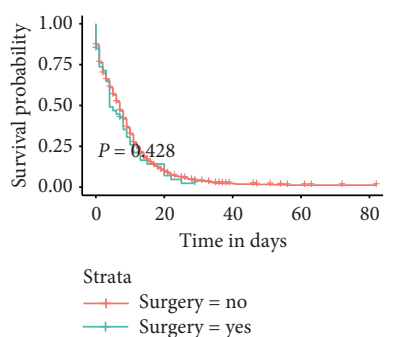

(a)

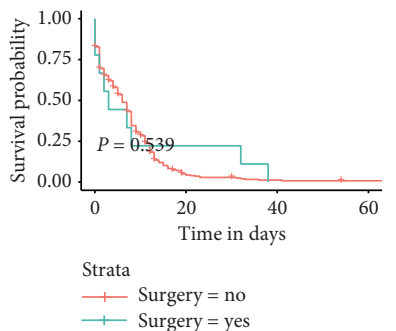

(e)

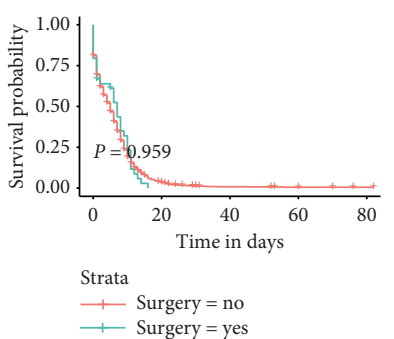

(b)

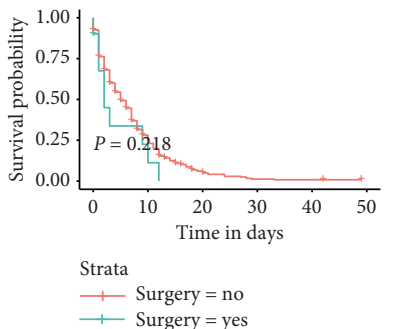

(f)

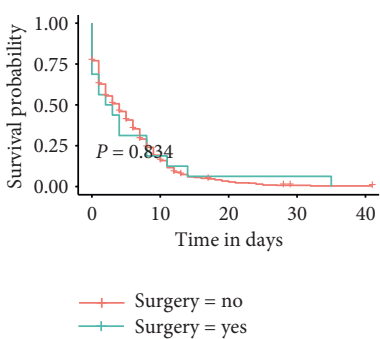

(c)

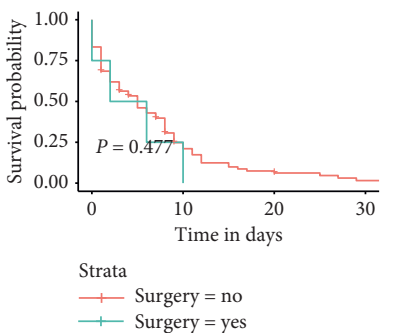

(g)

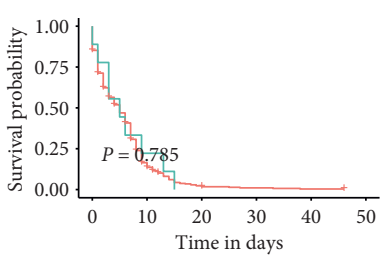

Strata

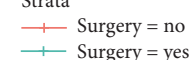

(d)

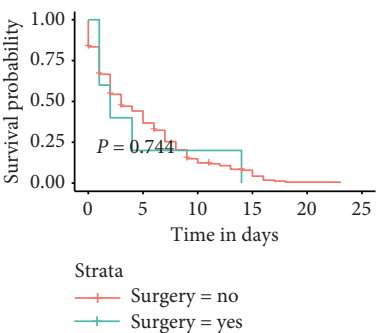

(h)

FIGURE 8: Kaplan-Meier survival curves and log-rank tests for each patient subgroup based on treatment modality. Differences in OS between patients who underwent surgery and those who did not in each subgroup. (a) Bone-only. (b) Bone and liver. (c) Bone, lung, and, liver. (d) Bone, liver, and brain. (e) Bone and lung. (f) Bone and brain. (g) Bone, lung, and brain. (h) Bone, lung, liver, and brain.

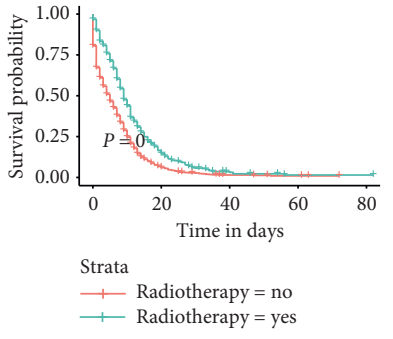

(a)

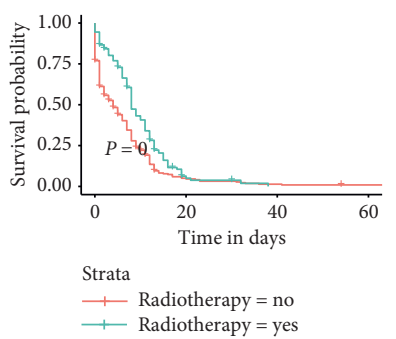

(e)

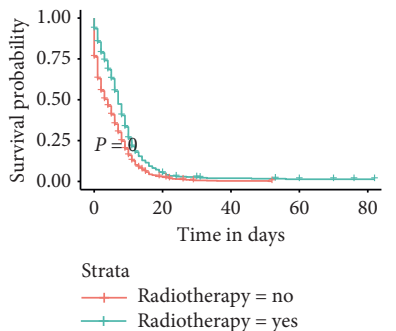

(b)

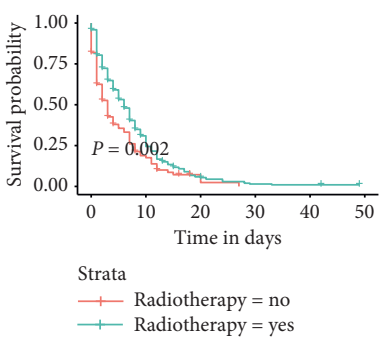

(f)

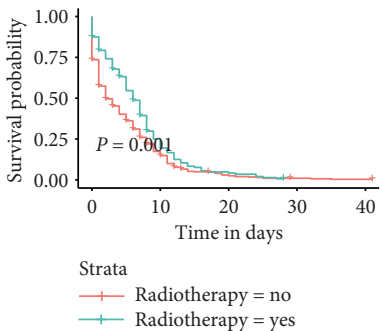

(c)



$$
\begin{aligned}
& \text { Strata } \\
& \square \text { Radiotherapy }=\text { no } \\
& \square \text { Radiotherapy }=\text { yes }
\end{aligned}
$$

$(\mathrm{g})$

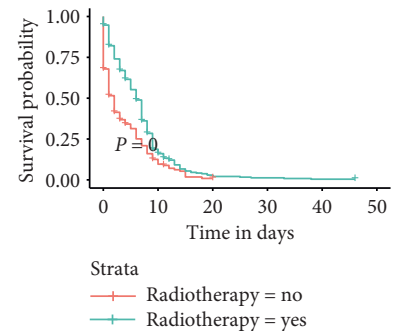

(d)



(h)

Figure 9: Kaplan-Meier survival curves and log-rank tests for each patient subgroup based on treatment modality. Differences in OS between patients who received radiotherapy and those who did not in each subgroup. (a) Bone-only. (b) Bone and liver. (c) Bone, lung, and, liver. (d) Bone, liver, and brain. (e) Bone and lung. (f) Bone and brain. (g) Bone, lung, and brain. (h) Bone, lung, liver, and brain.

increased risk may be associated with an increased prevalence of degenerative changes and comorbidities in various aspects of organ function [23]. In addition, older patients may be more sensitive to toxicity caused by systemic therapy, whereas younger patients are in good health and can better tolerate the side effects of chemotherapy and radiotherapy [24, 25]. Notably, unmarried status and male sex are poor prognostic factors in SCLC patients with BM. Unmarried patients do not receive psychological and financial support from their spouses, which results in a poorer prognosis [26].

The incidence of distant metastasis at the initial diagnosis of SCLC exceeds $60 \%$ [5]. The results of this study show that approximately $70 \%$ of SCLC patients initially diagnosed with BM have synchronous distant metastases from other sites. Previous studies that have reported that the liver is the most common organ for extraskeletal metastases, followed by the lung, are consistent with our 


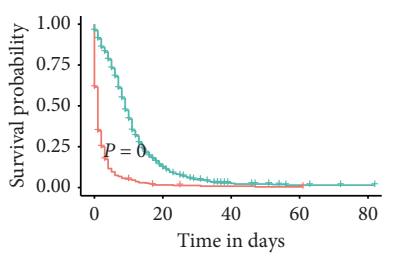

Strata

+ Chemotherapy $=$ no $\longrightarrow$ Chemotherapy $=$ yes

(a)

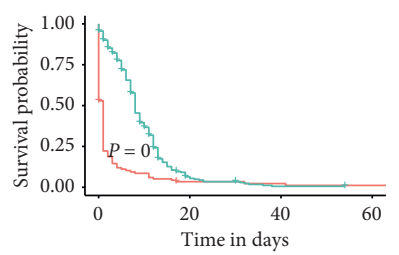

Strata

- Chemotherapy $=$ no

(e)

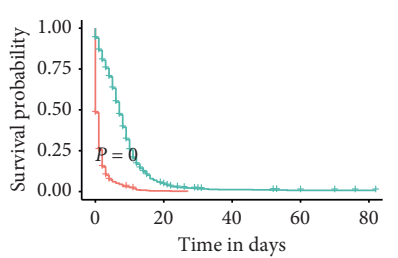

Strata

+ Chemotherapy $=$ no

$\_$Chemotherapy $=$yes

(b)

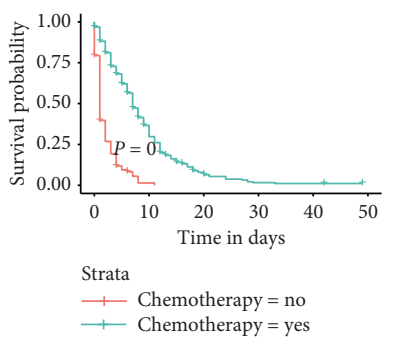

(f)

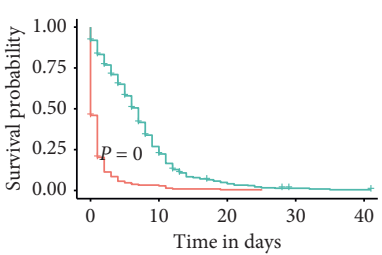

Strata

$\longrightarrow$ Chemotherapy $=$ no

Chemotherapy $=$ yes

(c)

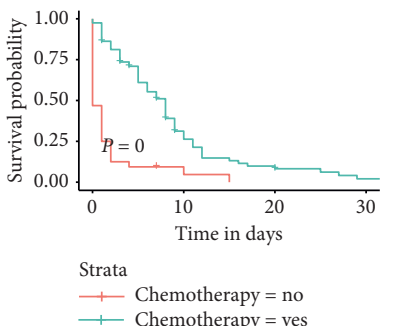

(g)

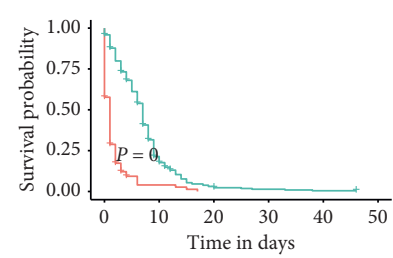

Strata

$\longleftarrow$ Chemotherapy $=$ no

(d)

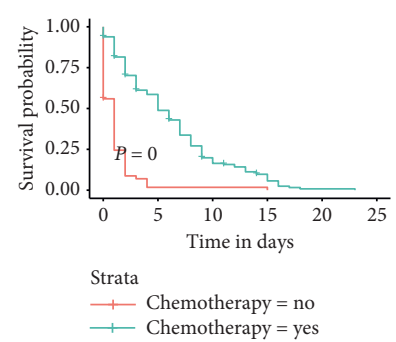

(h)

Figure 10: Kaplan-Meier survival curves and log-rank tests for each patient subgroup based on treatment modality. Differences in OS between patients who received chemotherapy and those who did not in each subgroup. (a) Bone-only. (b) Bone and liver. (c) Bone, lung, and, liver. (d) Bone, liver, and brain. (e) Bone and lung. (f) Bone and brain. (g) Bone, lung, and brain. (h) Bone, lung, liver, and brain.

results $[13,19]$. The incidence of liver metastasis was much higher in patients with newly diagnosed SCLC (17.5\%) than in patients with nonsmall cell lung cancer (4\%) [27]. Our results show that more than $50 \%$ of patients have liver metastasis combined with extraskeletal synchronous metastases. We also unexpectedly found that liver metastases were responsible for most of the multisite metastases. This may be explained by the finding that since the liver is an immunosuppressive organ when liver metastases occur, this impedes the liver's immune surveillance of other ongoing metastases [28]. SCLC is a very aggressive malignancy with ubiquitous organ metastases that progress from one organ to another in the majority of patients [29]. Thus, disease progression often leads to an increase in metastatic and a poor prognosis. Liver metastases significantly shorten the survival of lung cancer patients with BM [30]. Our study confirms the findings that liver metastases are always associated with poorer OS. In addition, whenever patients have liver metastases, there is a tendency for a worse prognosis regardless of the number of metastases at other sites, which is in line with previous reports [31]. It is also easy to observe that as the number of metastatic sites outside the bone increases, patients tend to exhibit a shorter survival time (Table 3 and Figure 7) $[30,31]$. These results suggest that we need to carefully evaluate the synchronous metastases in extraskeletal organs before treatment is initiated in SCLC patients with BM at initial diagnosis, resulting in more rational and effective treatment decisions.

The main treatment options for SCLC include surgery, chemotherapy, and radiotherapy [32]. The importance of surgery in the treatment of early stage SCLC is widely recognized, but surgery is often not recommended for patients with advanced disease. A growing body of research suggests that surgery is beneficial in prolonging the OS of patients with advanced SCLC $[33,34]$. Interestingly, in our study, surgery did not reduce the risk of death according to a multivariate Cox analysis that was performed for all patients and each patient subgroup. For patients with advanced SCLC, either chemotherapy alone or chemotherapy combined with radiotherapy is considered the standard treatment [35]. According to the Cox analysis of all patients, chemotherapy and radiotherapy effectively reduced the risk of death, with HRs of 0.272 and 0.801, respectively. All patients were stratified according to the sites of metastasis and treatment modality to further assess the survival benefit of the treatment modality. In the subgroup analysis, chemotherapy was found to be an independent prognostic factor for each subgroup, whereas radiotherapy showed a positive effect on prognosis for all subgroups except for the bone and brain metastases and bone, lung, and brain metastases subgroups. Through a subgroup analysis performed after the stratification of patients according to treatment modality and metastatic site, we once again confirmed the positive effect of chemotherapy and radiotherapy on prognosis and screened out subgroups of patients whose survival did not improve after radiotherapy. This is important to select a more precise treatment for patients, avoid wasting healthcare resources and guide clinicians in their treatment decisions.

Similar to other studies that have used the SEER database as a data source, this study inevitably has some limitations. First, only specific information on the four metastatic sites was included, and details of metastases were lacking, such as the number of metastatic foci and the sequence in which the organs became metastatic. Second, all metastasis-related information begins with the initial diagnosis, and follow-up 
information is lacking. Third, the SEER database does not record details of surgical, radiotherapy, and chemotherapy treatments (i.e., surgical procedures, radiation doses, chemotherapy regimens, and chemotherapy sequences). Fourth, the SEER database lacks biomarker information that may be prognostically relevant.

\section{Conclusions}

In summary, this study found that age, sex, primary site, radiotherapy, chemotherapy, brain metastasis, liver metastasis, and marital status influenced the OS of SCLC patients with $\mathrm{BM}$ at initial diagnosis. In addition, we constructed a nomogram model for predicting OS. When confronted with an individualized consultation, the nomogram can provide patients with relevant prognostic information and can enhance each patient's prognosis-based decision-making, which is important for the improvement of patient outcomes.

\section{Abbreviations}

SCLC: Small cell lung cancer

BM: Bone metastasis

SEER: Surveillance, Epidemiology, and End results

OS: Overall survival

ROC: Receiver operating characteristic

AUC: Area under the curve

DCA: Decision curve analysis.

\section{Data Availability}

The dataset from the SEER database generated and/or analyzed during the current study is available in the SEER dataset repository (https://seer.cancer.gov/).

\section{Ethical Approval}

Not applicable, as the study is based on a publicly available dataset.

\section{Consent}

Not applicable.

\section{Conflicts of Interest}

The authors declare that they have no conflicts of interest.

\section{Authors' Contributions}

ZY F and H S conceived, designed, and supervised the study. $\mathrm{ZH} \mathrm{H}$ and YX T performed the literature search. ZZ and XH $\mathrm{H}$ generated the figures and tables. $\mathrm{ZY} \mathrm{F}$ and $\mathrm{HZ} \mathrm{H}$ analyzed the data. ZY F wrote the manuscript and H S critically reviewed the manuscript. All authors have read and approved the manuscript.

\section{References}

[1] R. L. Siegel, K. D. Miller, and A. Jemal, "Cancer statistics, 2020," CA: A Cancer Journal for Clinicians, vol. 70, no. 1, pp. 7-30, 2020.

[2] R. Govindan, N. Page, D. Morgensztern et al., "Changing epidemiology of small-cell lung cancer in the United States over the last 30 years: analysis of the surveillance, epidemiologic, and end results database," Journal of Clinical Oncology, vol. 24, no. 28, pp. 4539-4544, 2006.

[3] C. M. Rudin, N. Ismaila, C. L. Hann et al., "Treatment of small-cell lung cancer: American society of clinical oncology endorsement of the American college of chest physicians guideline," Journal of Clinical Oncology, vol. 33, no. 34, pp. 4106-4111, 2015.

[4] Z. Sun, J. Chen, J. Aakre et al., "Genetic variation in glutathione metabolism and DNA repair genes predicts survival of small-cell lung cancer patients," Annals of Oncology, vol. 21, no. 10, pp. 2011-2016, 2010.

[5] M. Riihimäki, A. Hemminki, M. Fallah et al., "Metastatic sites and survival in lung cancer," Lung Cancer, vol. 86, no. 1, pp. 78-84, 2014.

[6] J. P. Van Meerbeeck, D. A. Fennell, and D. K. De Ruysscher, "Small-cell lung cancer," The Lancet, vol. 378, no. 9804, pp. 1741-1755, 2011.

[7] G. Selvaggi and G. V. Scagliotti, "Management of bone metastases in cancer: a review," Critical Reviews in Oncology/ hematology, vol. 56, no. 3, pp. 365-378, 2005.

[8] I. S. Bhattacharya and P. J. Hoskin, "Stereotactic body radiotherapy for spinal and bone metastases," Clinical Oncology, vol. 27, no. 5, pp. 298-306, 2015.

[9] D. Santini, S. Barni, S. Intagliata et al., "Natural history of nonsmall-cell lung cancer with bone metastases," Scientific Reports, vol. 5, no. 1, p. 18670, 2015.

[10] M. Kuchuk, I. Kuchuk, E. Sabri, B. Hutton, M. Clemons, and P. Wheatley-Price, "The incidence and clinical impact of bone metastases in non-small cell lung cancer," Lung Cancer, vol. 89, no. 2, pp. 197-202, 2015.

[11] D. Pruksakorn, A. Phanphaisarn, J. Settakorn et al., "Prognostic score for life expectancy evaluation of lung cancer patients after bone metastasis," Journal of Bone Oncology, vol. 10, pp. 1-5, 2018.

[12] A. Tsuya, T. Kurata, K. Tamura, and M. Fukuoka, "Skeletal metastases in non-small cell lung cancer: a retrospective study," Lung Cancer, vol. 57, no. 2, pp. 229-232, 2007.

[13] L. Gong, L. Xu, Z. Yuan, Z. Wang, L. Zhao, and P. Wang, "Clinical outcome for small cell lung cancer patients with bone metastases at the time of diagnosis," Journal of Bone Oncology, vol. 19, p. 100265, 2019.

[14] H. B. Burke, "Outcome prediction and the future of the TNM staging system," JNCI Journal of the National Cancer Institute, vol. 96, no. 19, pp. 1408-1409, 2004.

[15] Z. Ahmed, L. Kujtan, K. F. Kennedy, J. R. Davis, and J. Subramanian, "Disparities in the management of patients with stage I small cell lung carcinoma (SCLC): a surveillance, epidemiology and end results (SEER) analysis," Clinical Lung Cancer, vol. 18, no. 5, pp. e315-e325, 2017.

[16] M. Wolf, R. Holle, K. Hans, P. Drings, and K. Havemann, "Analysis of prognostic factors in 766 patients with small cell lung cancer (SCLC): the role of sex as a predictor for survival," British Journal of Cancer, vol. 63, no. 6, pp. 986-992, 1991.

[17] R. L. Camp, M. Dolled-Filhart, and D. L. Rimm, "X-tile: a new bio-informatics tool for biomarker assessment and outcomebased cut-point optimization," Clinical Cancer Research: an 
Official Journal of the American Association for Cancer Research, vol. 10, no. 21, pp. 7252-7259, 2004.

[18] J. Ranstam and J. A. Cook, "Kaplan-Meier curve," British Journal of Surgery, vol. 104, no. 4, p. 442, 2017.

[19] E. J. Kang, S. Y. Lee, H. J. Kim et al., "Prognostic factors and skeletal-related events in patients with small cell lung cancer with bone metastases at the time of diagnosis," Oncology, vol. 90, no. 2, pp. 103-111, 2016.

[20] J. Liu, H. Zhou, Y. Zhang et al., "Cause-specific death assessment of patients with stage I small-cell lung cancer: a competing risk analysis," Future Oncology, vol. 15, no. 21, pp. 2479-2488, 2019.

[21] Y. Wang, Z. Pang, X. Chen, T. Yan, J. Liu, and J. Du, "Development and validation of a prognostic model of resectable small-cell lung cancer: a large population-based cohort study and external validation," Journal of Translational Medicine, vol. 18 , no. 1, p. 237, 2020.

[22] J. D. Lara, A. Brunson, J. W. Riess, K. Kelly, P. N. Lara, and D. R. Gandara, "Clinical predictors of survival in young patients with small cell lung cancer: results from the California cancer registry," Lung Cancer, vol. 112, pp. 165-168, 2017.

[23] M. J. Aarts, J. G. Aerts, B. E. Van Den Borne, B. Biesma, V. E. P. P. Lemmens, and J. S. Kloover, "Comorbidity in patients with small-cell lung cancer: trends and prognostic impact," Clinical Lung Cancer, vol. 16, no. 4, pp. 282-291, 2015.

[24] R. C. Chen, T. J. Royce, M. Extermann, and B. B. Reeve, "Impact of age and comorbidity on treatment and outcomes in elderly cancer patients," Seminars in Radiation Oncology, vol. 22, no. 4, pp. 265-271, 2012.

[25] A. Gregor, P. Drings, M. Rinaldi et al., "Acute toxicity of alternating schedule of chemotherapy and irradiation in limited small-cell lung cancer in a pilot study (08877) of the EORTC lung cancer cooperative group," Annals of Oncology, vol. 6, no. 4, pp. 403-405, 1995.

[26] A. A. Aizer, M.-H. Chen, E. P. McCarthy et al., "Marital status and survival in patients with cancer," Journal of Clinical Oncology, vol. 31, no. 31, pp. 3869-3876, 2013.

[27] K. Kagohashi, H. Satoh, H. Ishikawa, M. Ohtsuka, and K. Sekizawa, "Liver metastasis at the time of initial diagnosis of lung cancer," Medical Oncology, vol. 20, no. 1, pp. 25-28, 2003.

[28] B. Ham, N. Wang, Z. D’Costa et al., “TNF receptor-2 facilitates an immunosuppressive microenvironment in the liver to promote the colonization and growth of hepatic metastases," Cancer Research, vol. 75, no. 24, pp. 5235-5247, 2015.

[29] A. Oikawa, H. Takahashi, H. Ishikawa, K. Kurishima, K. Kagohashi, and H. Satoh, "Application of conditional probability analysis to distant metastases from lung cancer," Oncology Letters, vol. 3, no. 3, pp. 629-634, 2012.

[30] H. Sugiura, K. Yamada, T. Sugiura, T. Hida, and T. Mitsudomi, "Predictors of survival in patients with bone metastasis of lung cancer," Clinical Orthopaedics \& Related Research, vol. 466, no. 3, pp. 729-736, 2008.

[31] H. Cai, H. Wang, Z. Li, J. Lin, and J. Yu, "The prognostic analysis of different metastatic patterns in extensive-stage small-cell lung cancer patients: a large population-based study," Future Oncology, vol. 14, no. 14, pp. 1397-1407, 2018.

[32] G. P. Kalemkerian and B. J. Schneider, "Advances in small cell lung cancer," Hematology/oncology Clinics of North America, vol. 31, no. 1, pp. 143-156, 2017.

[33] A. Badzio, K. Kurowski, H. Karnickamlodkowska, and J. Jassem, "A retrospective comparative study of surgery followed by chemotherapy vs. non-surgical management in limited-disease small cell lung cancer," European Journal of Cardio-Thoracic Surgery, vol. 26, no. 1, pp. 183-188, 2004.

[34] W. Eberhardt, G. Stamatis, M. Stuschke et al., "Prognostically orientated multimodality treatment including surgery for selected patients of small-cell lung cancer patients stages IB to IIIB: long-term results of a phase II trial," British Journal of Cancer, vol. 81, no. 7, pp. 1206-1212, 1999.

[35] G. P. Kalemkerian, W. Akerley, P. Bogner et al., "Small cell lung cancer," Journal of the National Comprehensive Cancer Network, vol. 11, no. 1, pp. 78-98, 2013. 